\title{
Limits on Low-Mass WIMP Dark Matter with an Ultra-Low-Energy Germanium Detector at $220 \mathrm{eV}$ Threshold
}

\author{
Shin-Ted Lin ${ }^{a}$, Muhammed Deniz ${ }^{* a, b}$, Henry T. Wong ${ }^{a}$ (for TEXONO Collaboration) \\ ${ }^{a}$ Institute of Physics, Academia Sinica, Taipei 11529, Taiwan. \\ ${ }^{b}$ Department of Physics, Middle East Technical University, Ankara 06531, Turkey. \\ E-mail: htwongephys.sinica.edu.tw
}

\begin{abstract}
The theme of the TEXONO research program is on the studies of low energy neutrino and dark matter physics. The current goals of the TEXONO research program are on the development of germanium detectors with sub-keV sensitivities to realize experiments on neutrino magnetic moments, neutrino-nucleus coherent scattering, as well as WIMP dark matter searches. An energy threshold of $220 \mathrm{eV}$ was achieved with a four-channel ultra-low-energy germanium prototype detector each with an active mass of $5 \mathrm{~g}$ at the Kuo-Sheng Neutrino Laboratory. New limits were placed for the couplings of low-mass WIMPs with matter. Data are being taken with a $500 \mathrm{~g}$ Point Contact Germanium detector, where a threshold of $\sim 350 \mathrm{eV}$ was demonstrated. The dark matter program will evolve into a dedicated experiment at an underground laboratory under construction in Sichuan, China.
\end{abstract}

The 2009 Europhysics Conference on High Energy Physics,

July 16 - 222009

Krakow, Poland

\footnotetext{
* Speaker.
} 


\section{Introduction and Highlights}

A research program on low energy neutrino and dark matter physics is pursued at the KuoSheng Neutrino Laboratory (KSNL) by the TEXONO Collaboration[1]. The laboratory is located at a distance of $28 \mathrm{~m}$ from a $2.9 \mathrm{GW}$ reactor core and has an overburden of about 30 meterwater-equivalent. Results on neutrino magnetic moments[2] and neutrino-electron scattering crosssection have been obtained[3]. The present goals are to develop advanced detectors with kg-size target mass, $100 \mathrm{eV}$-range threshold and low-background specifications [ $₫$ ] for the searches of Weakly Interacting Massive Particles (WIMPs)[5] at the low-mass region as well as the studies of neutrinonucleus coherent scattering[6] and neutrino magnetic moments.

\section{Results on Dark Matter Searches}

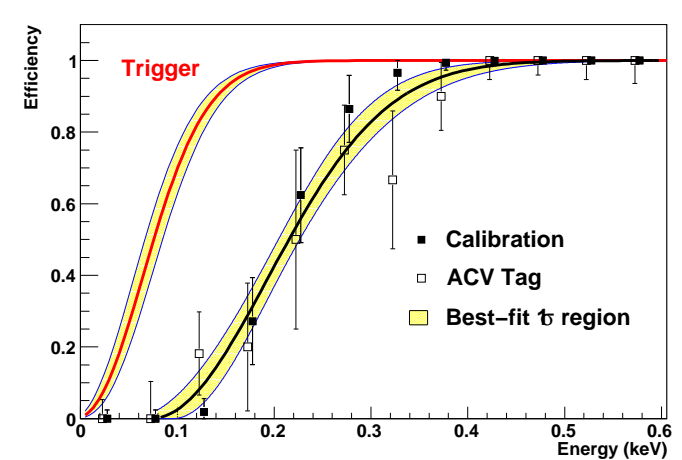

Figure 1: The trigger efficiency for physics events recorded by the DAQ system and analysis efficiency of the PSD cut with the best-fit $1 \sigma$ region, using the $20 \mathrm{~g}$ ULEGe prototype detector, as derived by the the ${ }^{55} \mathrm{Fe}$-calibration and in situ background events with ACV tags, respectively.

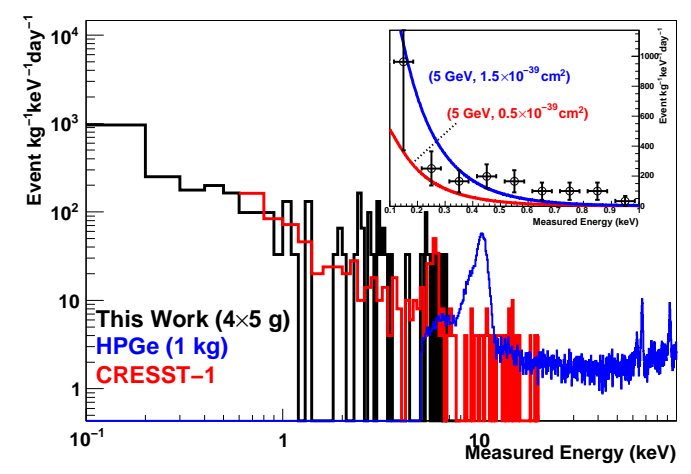

Figure 2: The measured spectrum of ULEGe with $0.338 \mathrm{~kg}$-day of data, after various background suppression procedures. Background spectra of the CRESST-I experiment[7] and the HPGe[2] are overlaid for comparison. The expected spectra for two cases of $\left(\mathrm{m}_{\chi}, \sigma_{\chi \mathrm{N}}^{\mathrm{SI}}\right)$ are superimposed onto the inset.

A four-channel Ultra-Low-Energy Germanium (ULEGe) prototype detector with a total active mass of $20 \mathrm{~g}$ has collected low-background data at KSNL[5]. The trigger and analysis efficiencies are shown in Figure 1. An energy threshold of $(220 \pm 10) \mathrm{eV}$ was achieved at an efficiency of $50 \%$. The background spectrum with $0.338 \mathrm{~kg}$-day of exposure is displayed in Figure 2. Constraints on WIMP-nucleon spin-independent $\left[\sigma_{\chi N}^{\mathrm{SI}}\right]$ and spin-dependent $\left[\sigma_{\chi N}^{\mathrm{SD}}(\mathrm{n})\right]$ couplings as functions of WIMP-mass $\left(\mathrm{m}_{\chi}\right)$ were derived, as depicted in Figures B \&\&, respectively. Overlaid on the plots are results from experiments which define the current exclusion boundaries, the DAMA-allowed regions and that favored by SUSY models[5, 7]. The KSNL limits improve over previous results at $\mathrm{m}_{\chi} \sim 3-6 \mathrm{GeV}$. Sensitivities for full-scale experiments at $1 \mathrm{cpd}$ background level are projected as dotted lines. The observable nuclear recoils at $\mathrm{m}_{\chi}=5 \mathrm{GeV}$ and $\sigma_{\chi \mathrm{N}}^{\mathrm{SI}}=0.5 \times 10^{-39} \mathrm{~cm}^{2}$ (allowed) and $1.5 \times 10^{-39} \mathrm{~cm}^{2}$ (excluded) are superimposed with the measured spectrum in the inset of Figure 2 for illustrations. 


\section{Performance of Point-Contact Germanium Detectors}

The design of Point-Contact Germanium (PCGe) detectors was first proposed in the 1980's [8], offering the potential merits of sub-keV sensitivities with $\mathrm{kg}$-scale target mass. There are intense recent interest triggered by successful realization and demonstration of the detector technique [9]. A PCGe of target mass $500 \mathrm{~g}$ was constructed and has been collecting data in KSNL since early 2009.

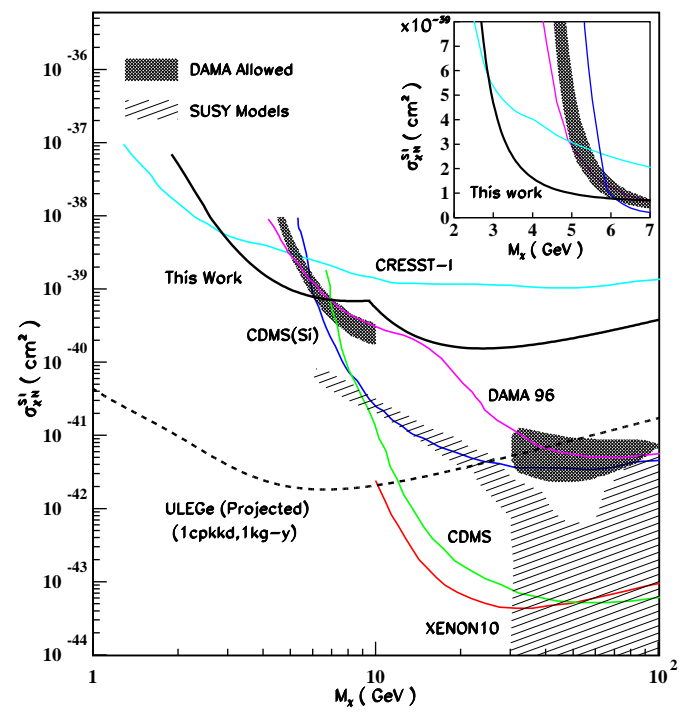

Figure 3: Exclusion plot of the spin-independent $\chi \mathrm{N}$ cross-section versus WIMP-mass.

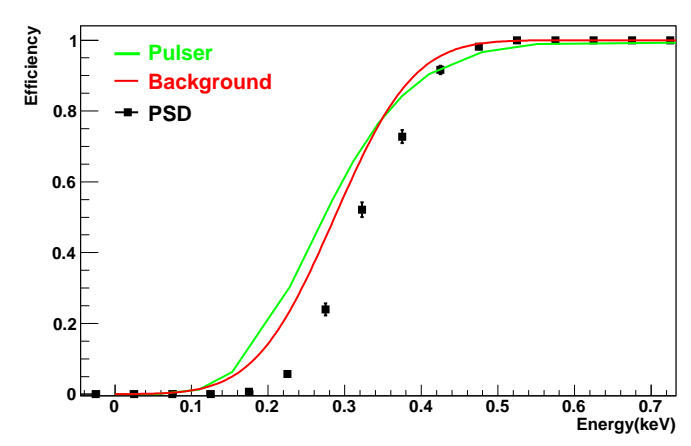

Figure 5: The trigger and analysis efficiencies of the $500 \mathrm{~g}$ PCGe detector, as derived from the test pulser and in situ events, respectively.

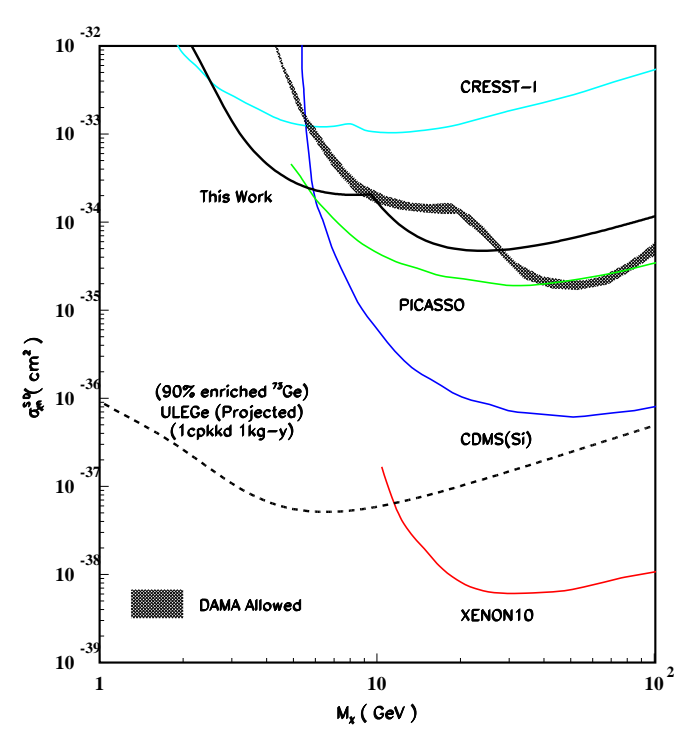

Figure 4: Exclusion plot of the spin-dependent $\chi$-neutron cross-section versus WIMP-mass.

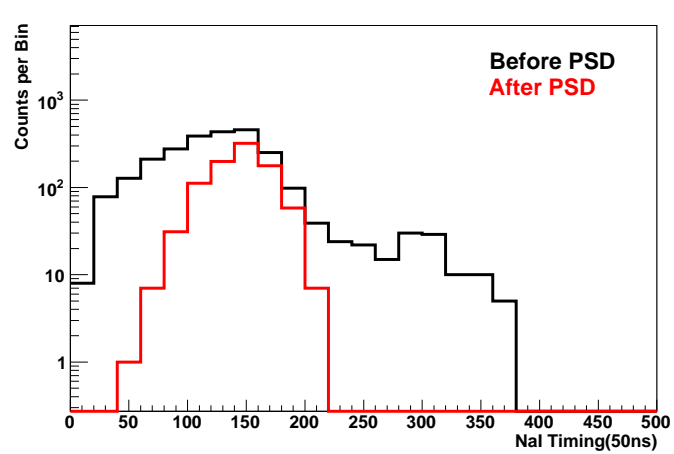

Figure 6: Events as a function of relative timing between $\mathrm{ACV}-\mathrm{NaI}(\mathrm{Tl})$ and PCGe systems, before and after PSD selection.

Similar procedures to those developed for the ULEGe were adopted to study the efficiency factors below the electronic noise edge. The results, analogous to those of Figure 1, are displayed in Figure 5. The trigger efficiencies were measured with two methods. The fractions of calibrated pulser events above the discriminator threshold provided the first measurement, while the studies on the amplitude distributions of in situ data contributed to the other. The relative timing between the 
PCGe and anti-Compton (ACV) NaI(Tl) detectors is shown in Figure 6, for "sub-noise edge" events at 200-400 eV before and after the pulse shape discrimination (PSD) selection processes. Events in coincidence with ACV at the "50-200 ns" window are due to multiple Compton scatterings, which are actual physical processes having similar pulse shapes as the neutrino and WIMP signals. It can be seen that only these events have substantial probabilities of surviving the cuts, and the fractions constitute to the PSD efficiencies. The threshold at $\sim 50 \%$ combined efficiencies is $\sim 350 \mathrm{eV}$. Intensive background and optimization studies with the PCGe at KSNL are underway.

\section{Status and Plans}

An excellent candidate site for a deep underground laboratory was recently identified in Sichuan, China where the China Jin-Ping Laboratory (CJPL) is being constructed [10]. The laboratory has more than $2500 \mathrm{~m}$ of rock overburden, is accessible by a road tunnel built for public traffic, and is supported by excellent infrastructures already available near the entrance. The first cavern of size $6 \mathrm{~m}$ (height)X6 m(width)X40 m(depth) is scheduled for completion in early 2010.

The low energy neutrino physics program will continue at KSNL, where a $900 \mathrm{~g}$ PCGe detector will be installed in 2010. Dedicated dark matter search with both $20 \mathrm{~g}$ ULEGe and $500 \mathrm{~g}$ PCGe detectors will be the first experimental program conducted at CJPL commencing 2010.

\section{References}

[1] H. T. Wong, Mod. Phys. Lett. A 19 (1207) 2004.

[2] H. B. Li et al., Phys. Rev. Lett. 90 (131802) 2003; H. T. Wong et al., Phys. Rev. D 75 (012001) 2007.

[3] H. B. Li et al., Nucl. Instrum. Methods A 459, (93) 2001; M. Deniz, H. T. Wong, [arXiv:0810.0809] 2008 ; M. Deniz, in proceedings of Mock conference, POS (EPS-HEP 2009) 285

[4] H. T. Wong, Mod. Phys. Lett. A 23 (1431) 2008.

[5] S. T. Lin et al., Phys. Rev. D 76 (061101 R) 2009 and references therein.

[6] H. T. Wong et al., J. Phys. Conf. Ser. 39 (266) 2006.

[7] M. Drees and G. Gerbier Phys. Lett. B 667 (241) 2008 and references therein.

[8] P.N. Luke et al., IEEE Trans. Nucl. Sci. 36 (926) 1989.

[9] P. A. Barbeau, J. I. Collar and O. Tench JCAP 09 (009) 2007.

[10] D. Normile, Science 324 (1246) 2009. 